\title{
Nonlinear dynamics and sustainable development of industrial enterprises within the framework of synergetic paradigm
}

\author{
Sergey Chuprov ${ }^{1, \mathrm{a}}$ \\ ${ }^{1}$ Baikal State University, 664003 Lenin St. 11, Irkutsk, Russia
}

\begin{abstract}
The paper considers development of industrial complexes in the context of synergetic paradigm. Contradictions and dynamism of the world and national economies have become the source of incredible changes interfering the work of industrial complexes. Russian industry suffers from the threats that are caused by resource degradation and ruin of the sustainability of industrial enterprises' work because of external factors. As a background to existing institutional changes in Russia and WTO, the entrance into the world economy globalization disturbs the work of enterprises, which, in turn, can initiate non-linear processes and as a result, fast and sharp reconstructions studied in synergetics. The influence of an information factor on the behavior of industrial enterprises and their stability in the environment of innovative changes and the economy of knowledge is discussed. The results of research obtained in the theory of nonlinear fluctuations are applied in the analysis of stability property in developing economic systems. Therefore, in terms of the catastrophe theory it is possible to discuss dynamic processes of economic evolution within activities of the developing industrial enterprises related to perestroikas and efficiency. It is shown that, from the point of view of the mathematical perestroikas theory, poorly developed enterprises with smaller losses pass into the improved stable state rather than a more advanced enterprises, which stability causes additional difficulties in acquiring a stable effective state. Thus, provided that there is a possible spasmodic, instead of continuous one, transition to the improved stable state, an enterprise will be able to evolve itself while approximating a similar state.
\end{abstract}

\section{Introduction}

Solution of a problem of maintaining the efficiency and stability of the developing industry enterprise during quick and sudden changes in the globalizing business environment has become of priority in the economic research recently. Against the dynamic background of institutional and innovative transformations being executed in Russia, the activities of industrial enterprises are subjected to risks of resource deterioration, competitive ability loss and sliding towards bankruptcy. On this account, it is an important challenge to develop and improve the control technologies for stability of enterprises in the context of systems analysis, management, cybernetics, etc. and of state-of-the-art knowledge of nonlinear dynamics and self-organization theory, which form the ground for the interdisciplinary synergetic paradigm.

Within the framework of its ideas, a logical explanation is given for the evolution of industrial enterprises from unstable equilibrium to stable nonequilibrium, as well as for qualitative modifications in their activities, in particular, those connected with transition from a stable and less effective state to a more effective one. Along with this, from the perspective of the entropy approach and information theory, it becomes possible to give reasons for the nonlinear (exponential) dependence of the industrial enterprise performance on the amount of accumulated information and to substantiate the conditions for maintaining the stability of this effect trends of change with regard to indicators observed.

\section{Materials and methods}

The concepts of the theory of systems, system analysis, cybernetics and synergetics have served as a basis for research methodology. Theoretical presentations of these sciences allow exposing and deeper understanding of the behavior and development of complex dynamical systems, their evolution and restructuring in the conditions of the changeable economic environment. In view of classic and modern concepts about the systems, generalization of the results obtained earlier is conducted in regard to complexity of the systems, their chaotic and ordered behavior. Within the bounds of entropy and synergetic

\footnotetext{
achuprov@isea.ru
} 
approaches the author analyzes reorganizations and operating efficiency of economic systems.

\section{Results and discussion}

Modern knowledge economy is formed against the background of accelerating innovational development of economic structures and extensive use of high technologies in production of goods and provision of services. Development prospects for production complexes are in line with the country's innovative modernization of the national economy aimed at generating design and technological knowledge, their materialization in science-intensive projects and developing the output of competitive products. The current problem is acquiring a dominant and urgent nature, also due to Russia's entering to the World Trade Organization, which results in increasing the risks of regional sales "compression" in regard to commodity producers and their loss of a number of profitable segments of the manufactured products market. Thus, the industry is now experiencing a whole spectrum of threats both from the globalizing world economy and internal production-technogical and financial-economic imbalances.

There arises a logical question about the effect of innovative development of production complexes, as perfection of their management systems, they are reaching, requires use of information and computer technologies, designing of algorithms and software products, and this is connected with additional costs that are covered by the revenues. The effectiveness of the system depends on its structure and cost, pay-offs and paybacks, while keeping in mind that stability of the enterprise's profit is provided by the aggregate of components of the management system. Modern researches confirm the fact that the effect of information sector's growth reflects laws of the transition to the information society and increase of attention to information area.

When making a comparative analysis of the management system effectiveness let us assume that its value is proportional to the amount of information that the system generates to suppress frictions violating stable activities of production complexes. Indeed, the increase in controlling information requires innovative modernization of the system, accumulation of its capacities and values (channels of data collection, storage facilities and processors for converting information, means of their display, and so on). Therefore, in order to assess the effectiveness of industrial enterprise's performance it is logical to make use the information criterion, proposed in the 60's of the last century by V.A. Trapeznikov [1].

In his mind, a part of the information is embodied in the objects of labor, the other part is accumulated in the form of knowledge, and, finally, part of it is consumed in the labour process in struggle against irregularity. In the economic system of the enterprise, information not only enters it, during management performance in the form of made decisions and reports, but it is also materialized in objects and means of labor in the form of knowledge embodied in them. According to the concept of V.A. Trapeznikov, the maximum values for the economic system effect are implemented only by a rapid increase in its value. Therefore, in the framework of the statistical approach, as it is shown by the following analysis, there is a mathematical relationship between the volume of source $I_{0}$ and of the accumulated $I$ information and the effect of functioning $E$ (in regard to maximum possible effect $E$ max ) in the economic system (Figure 1).

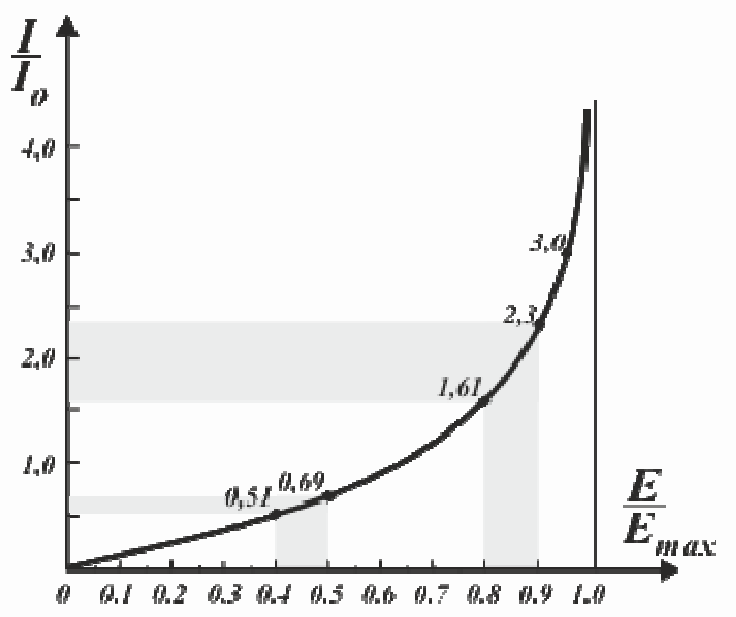

Fig. 1. Relationship between the relative values of the number of controlling information $I$ amounts and activities effect $E$ of the enterprise

As a result, maintenance of the stable level of economic effect $E$ in the field of large values makes the enterprise increase the payback period of its management system, and within the limits of approximating its effect to maximum $E$ max the payback period increases multifold. So, while modernizing the management system in order to increase effect level $E$ from 0.80 to 0.90 from maximum $E$ max, the payback period of the improved management system will increase by a factor of 1.71 in comparison with the increase of the effect from 0.70 to 0.80 from $E \max$. By a continuing increase of effect level $E$ for the enterprise from 0.90 to 0.95 payback, the period of its management will increase by 2.00 compared with the option of increasing the effect from 0.80 to 0.90 of maximum $E \max$.

The given comparative analysis of the management system effectiveness in regard to ensuring stable value $E$ of the economic effect of the enterprise resulted from the fact that in this case the effect value must be in the given interval from the lower to the upper limit. The author's calculations have confirmed that the increase of the level for a stable economic effect of the enterprise's activities from $0.80 \div 0.90$ to $0.90 \div 0.95$ from maximum limit $E$ max is reached only by increasing the payback period of the management system by a 
factor of $1.64 \div 2.03$ in comparison with a variant of the management system modernization, providing a transition from $0.70 \div 0.80$ to the range of $0.80 \div 0.90$ from maximum level $E$ max [2].

The analytical value of the information approach is that it allows identifying the existing regularities comprehensively and evaluating the amount of influence of the enterprise's knowledge on its development in the complex interaction of information, organizational, managerial, economic and other processes at the enterprise. The multifaceted development of this problem makes it possible to better understand the mechanism of an effective and stable performance of enterprises in the environment of innovative changes and the economy of knowledge.

The current theory of economic development embraces achievements from a broad range of branches of knowledge, and, primarily, those that address nonlinear processes in nature and in the society. This allows us to broaden our conceptual horizon of the innovative development of the region economic systems in terms of globalization and increasing competition on domestic and foreign markets.

On the one hand, the evolutionary systems typically have stable structures, but on the other hand, the destruction of the structure and the establishing another new structure are also typical. At the same time, the process of the system's evolution is cyclic in its nature and can be described as a series of smooth and abrupt phases.

The nonlinear dynamics shows this process as a series of alternating phases of order and chaos, based on the principle "development through instability". In the course of this process, chaos arises in a well-ordered system. As a result, under conditions of strong nonequilibrium, the system loses its stability; and, at the point of bifurcation, the system, which is enveloped in chaos, (under the impact of small disturbances) cardinally changes direction of its development, and order is restored again. Then, chaos arises and develops again, and the system evolves in the same way. Here the necessary prerequisite for the system's instability is the fact that it exchanges the flows of materials, energy or information with its environment; which, in its turn, allows the external disturbances to disturb the system from state of equilibrium and, from time to time, to "keep" it in unstable state.

In the catastrophe theory the loss of stability due to a change of the system's parameter is called either bifurcation of state of equilibrium or a spontaneous process. As the parameter is getting closer to the bifurcation value, the system loses its equilibrium, moving to another equilibrium position, or there arises a pair of equilibria. In this case, of the two states that appear or disappear, one is stable, the other is unstable.

In the author's opinion, this scenario is typical of the initial stage of an enterprise's crisis. At this stage, the system tries to retain its former stability of resource sharing with its environment. However, under the influence of disturbances, the enterprise's equilibrium is being undermined and its stability is decaying. The mounting crisis pushes the enterprise off its quasiequilibrium, and its system has to "grope for" a new state close to that of equilibrium. But the resource base of the enterprise has already been undermined (wornout fixed assets and depletable circulating funds, conflicting staff, and so on) and as a result, it becomes unstable.

Further on, "if a stable equilibrium state describes the established conditions in some real system (say in economics, ecology or chemistry)," - V.Arnold explains, - "then when it merges with an unstable equilibrium state, the system must jump to a completely different state: as the parameter is changed, the equilibrium condition in the region under consideration suddenly disappears. It was jumps of this kind which led to the term catastrophe theory." [3]. It is clear that the further course of events in the enterprise can develop very rapidly, and the resolution of the crisis may have either an optimistic outcome (recovery of the resource potential, and, with it, of the quasi-equilibrium) or, more likely, a pessimistic one (wear and tear and bankruptcy).

Another scenario of loss of a stable equilibrium state has two options, with different phase portraits (state space) of the system. The first of these is a regeneration of equilibrium position into the limit cycle, i.e. the transition from a stable equilibrium to the unstable (Figure 2, top row). The second option is the dying away of the state of equilibrium in the unstable limit cycle, during which the cycle disappears, and after it the balance becomes unstable (Fig. 2, bottom row).
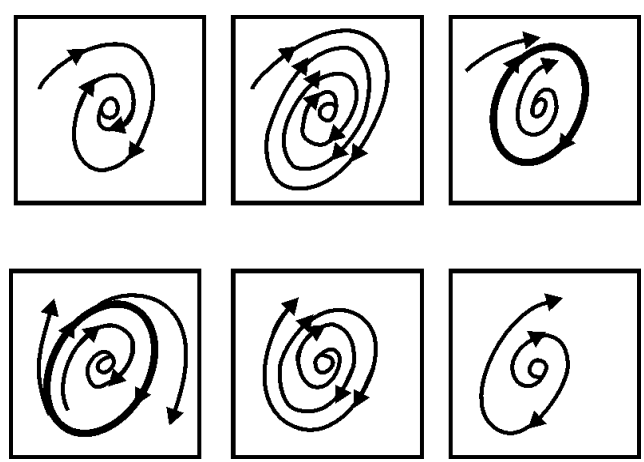

Fig. 2. Phase portraits of the system with loss of stability of stable equilibrium states [3]

The first option is more typical of a slow crisis in the enterprise: disruption of its reserve replacement and quasi-equilibrium goes gradually and slowly, but with each step it increases and "rocks" the system more drastically until its quasi-equilibrium changes from a stable to an unstable one. This gradual form of loss of stability is called mild which is seen from the oscillating behaviour for the equilibrium state (Figure 3, left). No wonder that this dynamic may fail to alarm analysts, who may still be confident of a stable behaviour of the enterprise. And only after some time the loss of stability would become obvious. 

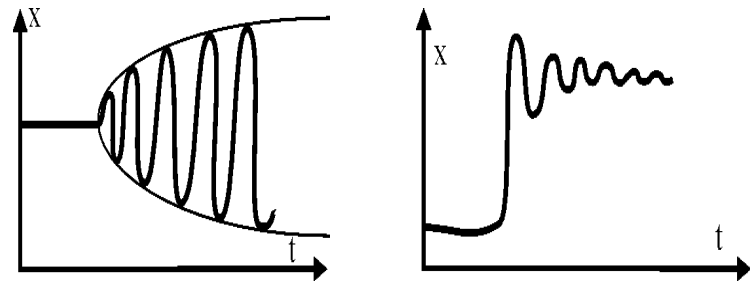

Fig. 3. Mild (on the left) and hard (on the right) loss of stability of the equilibrium state of the system [3]

The second case scenario, in comparison with the first one, is more typical of unstable work of the enterprise in high-risk conditions. At first, though being in crisis, the enterprise maintains its equilibrium, but eventually the cycling stops because of the deterioration of the circulation of resources between the enterprise and its environment. In the grip of the destructive dynamics, the system rapidly collapses; its quasiequilibrium loses its attraction and becomes unstable. Sharp loss of stability is called hard loss of stability. Here the system leaves its stationary state with a jump to a different state of motion (Fig 3, right).

At the same time, the next condition which establishes itself has little in common with equilibrium states or periodic oscillations, thus given the name of "strange attractor". This process is nothing else but dynamic chaos - non-periodic motion in a deterministic system. The transition from a stable equilibrium state to a strange attractor can take place both after hard and mild loss of stability. In the latter case, the cycle, thus created, then loses its stability. These non-periodic oscillations are very sensitive to small changes of the initial conditions, while at the same time the average characteristics of the behaviour are stable, the motion of such a system can be called turbulent. In other words, it is such a process that gives birth to lots of vortices of various sizes; due to this, their properties are influenced by chaotic fluctuations, and as a result they are changeable in space and random in time.

This "stall" of an economic system into a chaotic swirl mode can be explained by self-sustaining decomposition of the resource structure and increasing autonomism of its processes, which lead to chaotic behaviour of the system and disorganize its work on the whole. Arrhythmia of the resource flows (of raw materials, supplies, energy, goods, information, finances, etc.) creates chaos in production and management, deteriorates their co-ordination and paralyzes the work of the whole system. Homeostatic mechanism of the enterprise that was initially effective does not cope with external disturbances any more, and internal conflicts prevent adaptive forces from localizing focal points of crisis. The danger may appear when strong antagonists are in equilibrium, and the homeostat, when managerial decisions are delayed, can lose its stability, and there will be a displacement of the homeostatic equilibrium. As a result of the turbulent motion and decomposition of resources, the system can be pushed almost to the point of breakage, and the loss of its manageability and stable behaviour can be irreversible.
Taking into account the characteristics of nonlinear processes and the nature of the economic systems' development, let us use qualitative conclusions of the mathematical perestroikas theory that V. Arnold refers to [3]. Given the fact that the restructured economic system (in our case - an enterprise) is nonlinear and is in a "bad" stable-state condition, there are notable stages of its ascending development in order to move to a better stable-state condition. A visual representation of these stages is given in Figure 4, which allows us to use the following notation: $\mathrm{P}_{1}$ - the level of entrepreneurship and $\mathrm{P}_{2}$ - the level of economic efficiency of the system.

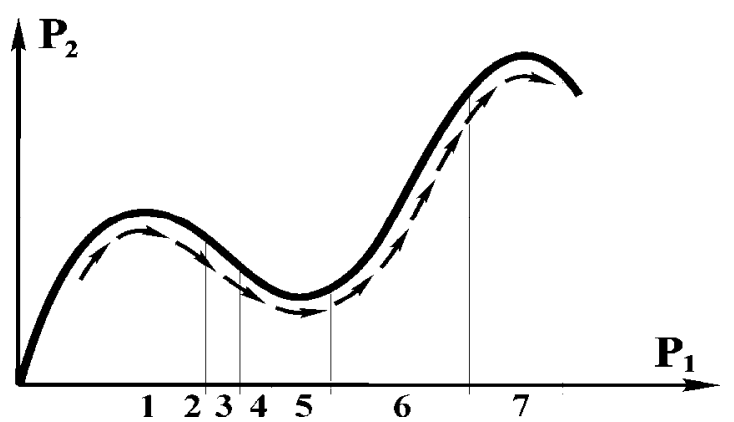

Fig. 4. Restructuring of the economic system from the perestroikas theory standpoint (by V. Arnold [3])

We characterize the stages of restructuring of the economic system, marked in Figure 4 on the abscissa axis with figures 1 to 7 :

- Stage 1. Being in a stable inefficient state, the enterprise has great difficulties in overcoming the resistance of the current organization of production and management, and begins to move to a higher level of economic efficiency of its operations;

- Stage 2. The enterprise's movement gathers head, but the action of conservative forces of its economic system (outdated infrastructure, lack of investments, the inertia of the company's management style, etc.) gains strength and the level of economic efficiency of the enterprise is markedly reduced;

- Stage 3. The enterprise's movement rate increases even further, but the influence of conservative forces of its economic system still affects it and even reaches its maximum; as a result, the level of economic efficiency of the enterprise continues to decrease;

- Stage 4. Before the level of economic efficiency of the enterprise becomes minimal, the resistance of its economic system turns weak, and after its complete disappearance, gives way to the positive scenario (upgrading and increasing the flexibility of the equipment, the introduction of high-tech industries, etc.) of the enterprise resource development.

- Stage 5. Inertia of deterioration of indices for the enterprise's economic efficiency ceases, they are at the lowest level; the trajectory of movement is at its pivot point; and when the positive forces start prevailing, the level of the enterprise's economic efficiency starts rising;

- Stage 6. The movement ("pulling") towards a better, stable state gathers head; capital improvements 
ensure a rapid growth of economic efficiency and a progressive development of the enterprise;

- Stage 7. The enterprise movement trajectory shows that its "climbing" to the peak of economic efficiency comes to its end; thereby the enterprise gains a stable state that differs from the initial state in its higher efficiency.

From the perestroikas theory standpoint, a lessdeveloped economic system gathers a better stable state, yielding lower losses than a developed one. The stability of the developed system runs it into additional trouble of gaining a stable efficient state. Moreover, if a jump, not a continuous transition, to a new, better stable state would be possible, then, when approaching it, the system would evolve ("gravitate") to it by itself.

\section{Conclusion}

Within the natural and economic scientific views on stability of dynamics the author suggests some lines for development of the methods and tools of cognition and provision of stability of the economic systems using the ideas of cybernetics, nonlinear dynamics, perestroikas theory [3-7].

The design of innovative development of industrial complexes reveals the nonlinear dependence of their functioning efficiency on the volume of useful information coming into their management system.

The analytical value of the information approach is that it allows identifying the existing regularities comprehensively and evaluating the amount of influence of the enterprise's knowledge on its development in the complex interaction of information, organizational, managerial, economic and other processes at the enterprise. The multifaceted development of this problem makes it possible to better understand the mechanism of an effective and stable performance of enterprises in the environment of innovative changes and the economy of knowledge. As a result, the significance of information for an enterprise increases with the increase of information capacity of production (correlation ratio is equal to 0,65 ) [8].

The current theory of economic development embraces achievements from a broad range of branches of knowledge, and, primarily, from those that address nonlinear processes in nature and in the society nonlinear dynamics, catastrophe theory, synergetics (for example [9-12]). This allows us to broaden our conceptual horizon of the innovative development of the economic systems in terms of globalization and increasing competition on domestic and foreign markets.

\section{Acknowledgment}

The work was realized under financial support of state task № 2014/52 for performing the state works in the area of science activity within the basic part of the project "Activation of Baikal Region Resource Potential as a Factor of its Steady Social-Economic Development” (№ 1841).

\section{References}

1. V.A. Trapeznikov, Upravlenie $i$ nauchnotekhnicheskii progress (Moscow, Nauka, 1983)

2. S.V. Chuprov, Upravlenie ustoichivost'yu proizvodstvennykh sistem: teoriya, metodologiya, praktika ( $2^{\text {th }}$ ed. Irkutsk, BGUEP, 2012)

3. V.I. Arnold, Teoriya katastrof $\left(3^{\text {th }}\right.$ ed. Moscow, Nauka, 1990)

4. J. Scheinkman, Econ. J., 100, (1990)

5. W.A. Brock, Nonlinearity and complex dynamics in economics and finance (Working papers, Wisconsin Madison - Social Systems, 1988)

6. E.G. Pavlidis, I. Paya, D.A. Peel, C. Siriopoulos. Eur. J. of Fin., 8, (2011)

7. A. Martynyuk, A. Mazko, S. Rasshyvalova, K. Teo, Non. Dyn. Syst. Theory, 11 (4), (2011)

8. Ye.V. Popov, Transakcii (Ekaterinburg, Ural'skoe otdelenie Rossijskoi Akademii Nauk, 2011)

9. M.P. Lazarević, Int. J. of Non-Lin. Mech., 73, (2015)

10. M. Bobrek, M. Sokovic, J. of Mat. Proc. Tech., 175, (2006)

11. S.X. Zeng, J.J. Shi, G.X. Lou, J. of Cl. Prod., 15, (2007)

12. T. Meynhardt, J.D Chandler, P. Strathoff, J. of Bus. Res. (2016) 